\title{
Coherence of temperature and velocity superstructures in turbulent Rayleigh-Bénard flow
}

\author{
Dominik Krug ${ }^{1, \dagger}$, Detlef Lohse ${ }^{1,2}$ and Richard J. A. M. Stevens ${ }^{1}$ \\ ${ }^{1}$ Physics of Fluids Group and Twente Max Planck Center, Department of Science and Technology, \\ Mesa+ Institute, and J. M. Burgers Center for Fluid Dynamics, University of Twente, \\ P.O. Box 217, 7500 AE Enschede, The Netherlands \\ ${ }^{2}$ Max Planck Institute for Dynamics and Self-Organization, Am Fassberg 17, 37077 Göttingen, Germany
}

(Received 26 August 2019; revised 13 December 2019; accepted 14 December 2019)

We investigate the interplay between large-scale patterns, so-called superstructures, in the fluctuation fields of temperature $\theta$ and vertical velocity $w$ in turbulent Rayleigh-Bénard convection at large aspect ratios. Earlier studies suggested that velocity superstructures were smaller than their thermal counterparts in the centre of the domain. However, a scale-by-scale analysis of the correlation between the two fields employing the linear coherence spectrum reveals that superstructures of the same size exist in both fields, which are almost perfectly correlated. The issue is further clarified by the observation that, in contrast to the temperature, and unlike assumed previously, superstructures in the vertical-velocity field do not result in a peak in the power spectrum of $w$. The origin of this difference is traced back to the production terms of the $\theta$ and $w$ variance. These results are confirmed for a range of Rayleigh numbers $R a=10^{5}-10^{9}$; the superstructure size is seen to increase monotonically with $R a$. Furthermore, the scale distribution of the temperature fluctuations in particular is pronouncedly bimodal. In addition to the large-scale peak caused by the superstructures, there exists a strong small-scale peak. This 'inner peak' is most intense at a distance of $\delta_{\theta}$ from the wall and is associated with structures of size $\approx 10 \delta_{\theta}$, where $\delta_{\theta}$ is the thermal boundary layer thickness. Finally, based on the vertical coherence relative to a reference height of $\delta_{\theta}$, a self-similar structure is identified in the velocity field (vertical and horizontal components) but not in the temperature.

Key words: turbulent convection, plumes/thermals, Bénard convection

\section{Introduction}

A remarkable feature of turbulent flows is that, amid the inherent disorder in both time and space, they frequently give rise to a surprisingly organized flow motion

$†$ Email address for correspondence: d.j.krug@utwente.nl 
on very large scales. Such very-large-scale structures in the fully turbulent regime have been reported, for example, for turbulent boundary layers (Hutchins \& Marusic 2007a), plane Couette flow (Lee \& Moser 2018) and Taylor-Couette turbulence (Huisman et al. 2014). Here we focus on superstructures in turbulent Rayleigh-Bénard convection (RBC), which is an idealized configuration that is widely used to study thermal convection (Ahlers, Grossmann \& Lohse 2009; Lohse \& Xia 2010; Chillà \& Schumacher 2012; Verma 2018). The strength of the non-dimensional thermal driving in RBC is given by the Rayleigh number $R a$, while the dimensionless heat transfer is characterized by the Nusselt number $\mathrm{Nu}$.

Large-scale organization in convective flows is widespread. An astonishing example is the formation of so-called cloud streets in the atmosphere that can extend for hundreds of kilometres (e.g. Young et al. 2002). Studying related features in RBC requires a cell with a large aspect ratio $\Gamma$. Naturally, this poses a challenge to experiments and simulations. Experimentally (e.g. Fitzjarrald 1976; Sun et al. 2005a,b; Zhou et al. 2012; Du Puits, Resagk \& Thess 2013; Hogg \& Ahlers 2013; Cierpka et al. 2019), it is very challenging to extract flow information beyond global parameters or local measurements of turbulence statistics. On the other hand, simulations for large aspect ratios are very costly if the thermal driving is sufficiently strong to achieve a moderately or even a strongly turbulent state. The first to tackle the problem numerically were Hartlep, Tilgner \& Busse (2003), and several related studies have since been presented in the literature (Parodi et al. 2004; Hartlep, Tilgner \& Busse 2005; Shishkina \& Wagner 2005, 2006, 2007; Von Hardenberg et al. 2008; Bailon-Cuba, Emran \& Schumacher 2010; Emran \& Schumacher 2015; Sakievich, Peet \& Adrian 2016). Very recently, the available ranges of large-aspect-ratio simulations have been extended significantly in Prandtl number $(P r)$ by Pandey, Scheel \& Schumacher (2018) and in $\Gamma$ as well as in $R a$ by Stevens et al. (2018).

From these papers, it has become clear that in RBC so-called superstructures exist at higher $\mathrm{Ra}$. These superstructures are significantly larger than the convection rolls at onset (see e.g. Drazin \& Reid (2004)) or as those in the weakly nonlinear regime (Morris et al. 1993). It is widely observed that the superstructure size increases with Ra (Fitzjarrald 1976; Hartlep et al. 2003; Pandey et al. 2018; Green et al. 2020), while the $\operatorname{Pr}$ dependence appears to be more complicated. For the latter, Pandey et al. (2018) report that at $R a=10^{5}$ the largest structures are found for $\operatorname{Pr} \approx 7$, but $\operatorname{Pr}$ variations over a significant range at higher $R a$ have not been reported yet. Stevens et al. (2018) showed that very large domain sizes up to $\Gamma=64$ are necessary to fully converge the size of the superstructures at $R a=10^{8}$. Finally, Von Hardenberg et al. (2008) and Pandey et al. (2018) demonstrate that superstructures evolve on time scales much longer than the free-fall time scale.

There is no consensus yet on how to best extract and quantify the superstructures in RBC. Researchers have relied on peaks in velocity and/or temperature power spectra (Von Hardenberg et al. 2008; Pandey et al. 2018; Stevens et al. 2018), velocity-temperature co-spectra (Fitzjarrald 1976; Hartlep et al. 2003; Green et al. 2020) or so-called integral length scales (Parodi et al. 2004; Stevens et al. 2018) to determine the structure size. A puzzling and as yet unexplained observation is that superstructures in the temperature $(\theta)$ field are larger than in the vertical-velocity $w$ field (Pandey et al. 2018; Stevens et al. 2018) when the structure size is determined based on the peaks in the power spectrum or the corresponding integral length scale. Also, visually, the difference between the temperature and vertical-velocity fields can easily be observed in the snapshots of the flow at mid-height, which are 

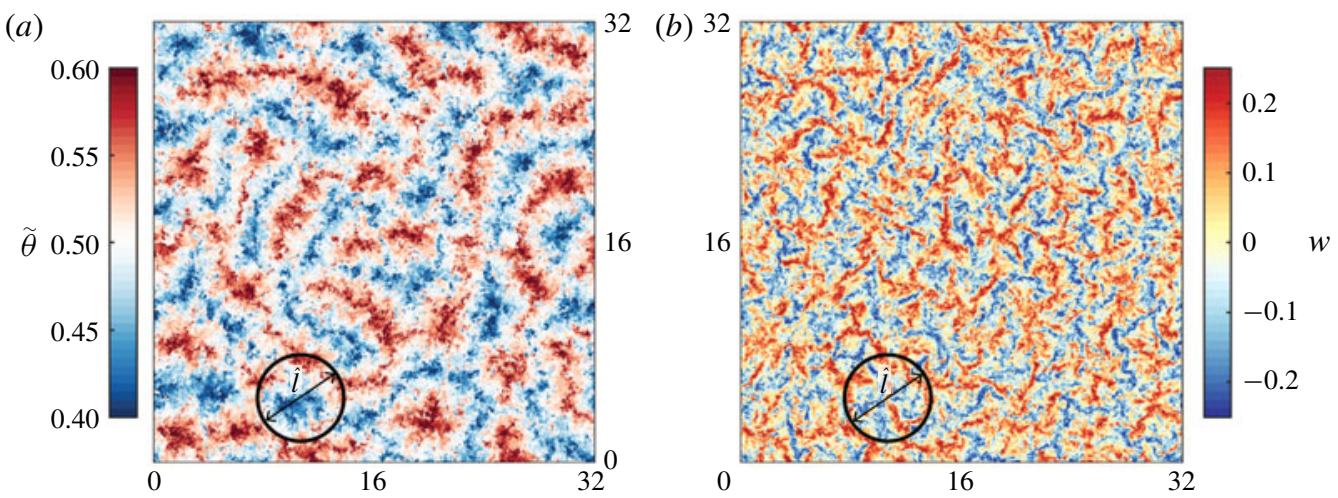

FIGURE 1. Snapshots of the temperature $(a)$ and the vertical-velocity $(b)$ field at midheight for a simulation in a $\Gamma=32$ cell with $\operatorname{Pr}=1$ at $R a=10^{8}$. The diameter of the circles in both panels indicates the superstructure size $\hat{l}=6.3$ (see table 1 ).

presented in figure 1. This figure reveals that the vertical-velocity field is dominated by significantly smaller structures than the temperature field. Moreover, the correlation between the two quantities appears much lower than one would naively expect, given that the temperature fluctuations provide the driving of $w$. These observations seem at odds with the notion that superstructures in RBC form large-scale convection rolls for which temperature and velocity scales should be of the same size.

To address and clarify this issue along with related questions, we use the dataset of Stevens et al. (2018) to assess energy distributions and coherence on a scale-byscale basis. Before presenting our results in $\S 3$, we provide the relevant details on the dataset of Stevens et al. (2018), together with the parameters of additional simulations performed for this study, in $\S 2$. We summarize our findings in $\S 4$.

\section{Dataset}

We solve the Boussinesq equations with the second-order staggered finite difference code AFiD. The code has been extensively validated and details of the numerical methods can be found in Verzicco \& Orlandi (1996), Stevens, Verzicco \& Lohse (2010), Stevens, Lohse \& Verzicco (2011), van der Poel et al. (2015) and Zhu et al. (2018). The governing equations in dimensionless form read:

$$
\begin{gathered}
\frac{\partial \boldsymbol{u}}{\partial t}+\boldsymbol{u} \cdot \nabla \boldsymbol{u}=-\nabla p+\sqrt{\frac{P r}{R a}} \nabla^{2} \boldsymbol{u}+\theta \hat{z}, \\
\nabla \cdot \boldsymbol{u}=0, \\
\frac{\partial \theta}{\partial t}+\boldsymbol{u} \cdot \nabla \theta=\frac{1}{\sqrt{\operatorname{RaPr}}} \nabla^{2} \theta,
\end{gathered}
$$

where $\hat{z}$ is the unit vector pointing in the opposite direction of gravity, $\boldsymbol{u}$ the velocity vector normalized by the free-fall velocity $\sqrt{g \alpha \Delta H}, t$ the dimensionless time normalized by $\sqrt{H /(g \alpha \Delta)}, \theta$ the temperature normalized by $\Delta$, and $p$ the pressure normalized by $g \alpha \Delta / H$. The control parameters of the system are $R a=\alpha g \Delta H^{3} /(\nu \kappa)$ and $\operatorname{Pr}=\nu / \kappa$, where $\alpha$ is the thermal expansion coefficient, $g$ the gravitational 


\begin{tabular}{lcrrrrrc}
$R a$ & $N_{x} \times N_{y} \times N_{z}$ & \multicolumn{1}{c}{$N u$} & \multicolumn{1}{c}{$R e_{h}$} & \multicolumn{1}{c}{$R e_{w}$} & \multicolumn{1}{c}{$\operatorname{Re}_{t}$} & $\hat{l}$ & $\delta_{\theta}=1 /(2 N u)$ \\
$1 \times 10^{5}$ & $2048 \times 2048 \times 64$ & 4.35 & 55.7 & 40.3 & 68.7 & 4.4 & 0.115 \\
$4 \times 10^{5}$ & $2048 \times 2048 \times 64$ & 6.48 & 111.7 & 84.3 & 140.0 & 4.5 & 0.077 \\
$1 \times 10^{6}$ & $3072 \times 3072 \times 96$ & 8.34 & 176.0 & 131.6 & 219.8 & 4.9 & 0.060 \\
$4 \times 10^{6}$ & $3072 \times 3072 \times 96$ & 12.27 & 349.7 & 250.8 & 430.4 & 5.4 & 0.041 \\
$1 \times 10^{7}$ & $4096 \times 4096 \times 128$ & 15.85 & 547.1 & 380.1 & 666.2 & 5.9 & 0.032 \\
$1 \times 10^{8}$ & $6144 \times 6144 \times 192$ & 30.94 & 1660.3 & 1056.1 & 1967.8 & 6.3 & 0.016 \\
$1 \times 10^{9}$ & $12288 \times 12288 \times 384$ & 61.83 & 4879.2 & 2962.3 & 5708.1 & 6.6 & 0.008
\end{tabular}

TABLE 1. The columns from left to right indicate the Rayleigh number, the numerical resolution in the horizontal and wall-normal directions $\left(N_{x} \times N_{y} \times N_{z}\right)$, the Nusselt number, and the horizontal $\left(R e_{h}=\sqrt{\left\langle v_{x}^{2}+v_{y}^{2}\right\rangle_{V}} \sqrt{\operatorname{Ra/Pr}}\right)$, vertical $\left(\operatorname{Re}_{w}=\sqrt{\left\langle w^{2}\right\rangle_{V}} \sqrt{\operatorname{Ra} / \operatorname{Pr}}\right)$ and total $\left(R e_{t}=\sqrt{\left\langle v_{x}^{2}+v_{y}^{2}+w^{2}\right\rangle_{V}} \sqrt{R a / P r}\right)$ Reynolds numbers. The length scale $\hat{l}$ denotes the superstructure scale based on the coherence spectrum $\gamma_{\theta w}^{2}$ (plotted as triangles in figure $8 b$ ) and $\delta_{\theta}$ is the thermal boundary layer thickness.

acceleration, $\Delta$ the temperature drop across the container, $H$ the height of the fluid domain, $v$ the kinematic viscosity, and $\kappa$ the thermal diffusivity of the fluid.

The boundary conditions on the top and bottom plates are no-slip for the velocity and constant for the temperature. Periodic conditions in the horizontal directions are used. In all our simulations, $\operatorname{Pr}$ is fixed to 1 and we analyse data for $\Gamma=L / H=32$, where $H$ is the vertical distance between the plates and $L$ the horizontal extension of the domain. Length scales are normalized by $H$ unless specified otherwise and we set $H=1$. Coordinates in the wall-parallel direction are denoted by $x$ and $y$ while the $z$-axis points along the wall-normal direction. Horizontal velocity components are denoted $v_{x}$ and $v_{y}$, respectively. A high spatial resolution in the boundary layer and bulk has been used to ensure that the resolution criteria set by Shishkina et al. (2010) and Stevens et al. (2010) are fulfilled. Details about the simulations can be found in table 1. The simulations for $R a=10^{8}$ and $R a=10^{9}$ have been reported before in Stevens et al. (2018), while the simulations for $10^{5} \leqslant R a \leqslant 10^{7}$ have been performed for this study. The horizontal, vertical and total Reynolds numbers indicated in table 1 represent the volume and time averages of $\operatorname{Re}_{h}=\left(\left\langle v_{x}^{2}+v_{y}^{2}\right\rangle_{V}\right)^{1 / 2}(\operatorname{Ra} / \operatorname{Pr})^{1 / 2}$, $R e_{w}=\left(\left\langle w^{2}\right\rangle_{V}\right)^{1 / 2}(\operatorname{Ra} / P r)^{1 / 2}$ and $\operatorname{Re}_{t}=\left(\left\langle v_{x}^{2}+v_{y}^{2}+w^{2}\right\rangle_{V}\right)^{1 / 2}(\operatorname{Ra} / \operatorname{Pr})^{1 / 2}$, respectively. In the following, we decompose instantaneous quantities $\tilde{\psi}$ into mean and fluctuating parts according to $\tilde{\psi}=\Psi+\psi$, where $\Psi=\langle\tilde{\psi}\rangle$ with $\langle\cdot\rangle$ denoting an average over a wall-parallel plane and time.

\section{Results}

In presenting our results, we initially $(\$ \S 3.1-3.3)$ restrict the discussion to a single Rayleigh number $\left(R a=10^{8}\right)$. A detailed discussion of the $R a$ dependence of our findings is then provided in $\$ 3.4$.

\subsection{Spectral distribution of energy and coherence of temperature and vertical velocity}

To evaluate the energy distribution across different scales, we first consider the one-sided power spectra $\Phi_{\psi \psi}(k)$, where $\psi$ is a zero-mean quantity (velocity or 


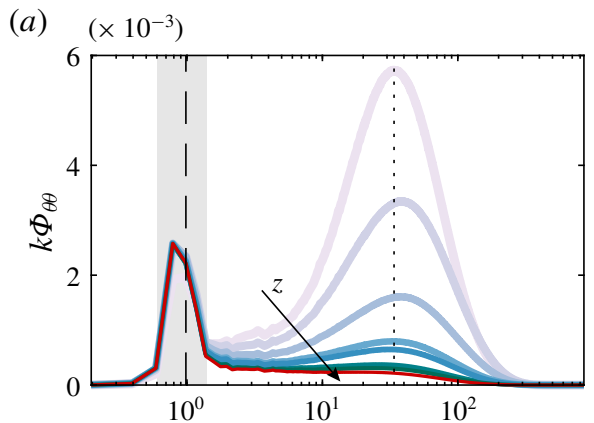

(b) $\left(\times 10^{-3}\right)$
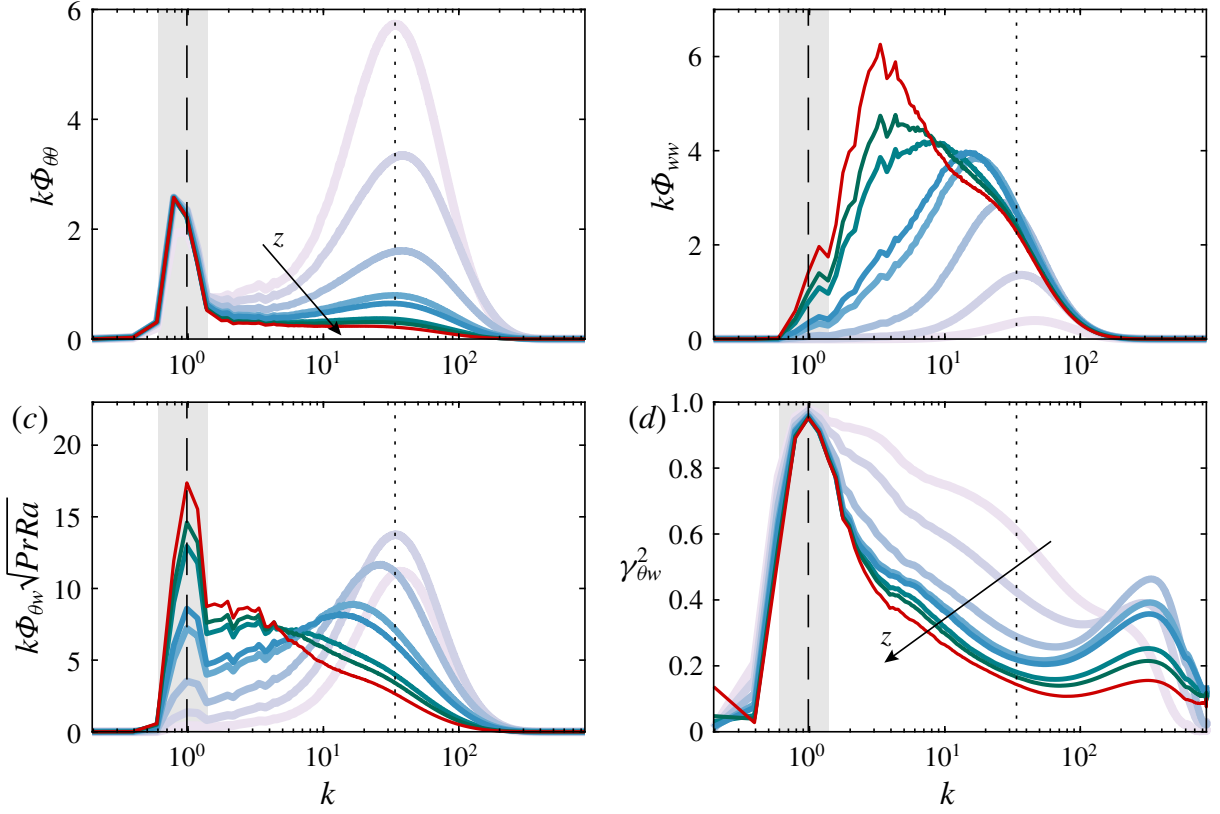

$z / H$

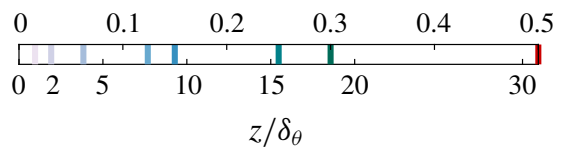

FIgURE 2. Premultiplied temperature $(a)$ and vertical-velocity $(b)$ power spectra. The premultiplied co-spectrum $k \Phi_{\theta w}(c)$ is normalized such that it integrates to the turbulent heat flux. $(d)$ Linear coherence spectrum $\gamma_{\theta w}^{2}$; see (3.2). The dashed and dotted vertical lines indicate $k=1$ and $k=34$, respectively. The grey shaded area marks the approximate range of superstructure scales $k=1 \pm 0.4$. The results presented here are computed for $R a=10^{8}$. The colour of the curves indicates the wall distance according to the legend at the foot of the figure.

temperature here) and $k$ is the radial wavenumber $k=\sqrt{k_{x}^{2}+k_{y}^{2}}$. The spectra are computed for horizontal planes and averaged in time. Results for $\theta$ and $w$ at several distances from the wall are presented in figure $2(a)$ and $(b)$, respectively. Data are presented in premultiplied form $k \Phi_{\psi \psi}$, such that the area under the curve equals the variance when plotted on a logarithmic scale, according to

$$
\left\langle\psi^{2}\right\rangle=\int_{0}^{\infty} \Phi_{\psi \psi} \mathrm{d} k=\int_{0}^{\infty} k \Phi_{\psi \psi} \mathrm{d}(\log k) .
$$

For reference, the wall-normal temperature and vertical-velocity variance profiles are presented in figures $3(a)$ and $3(b)$, respectively. The symbols in these figures mark the positions at which the spectra in figure 2 are computed.

First, we focus on the situation at mid-height $(z / H=0.5)$, which corresponds to the location of the snapshots shown in figure 1. These results are represented 

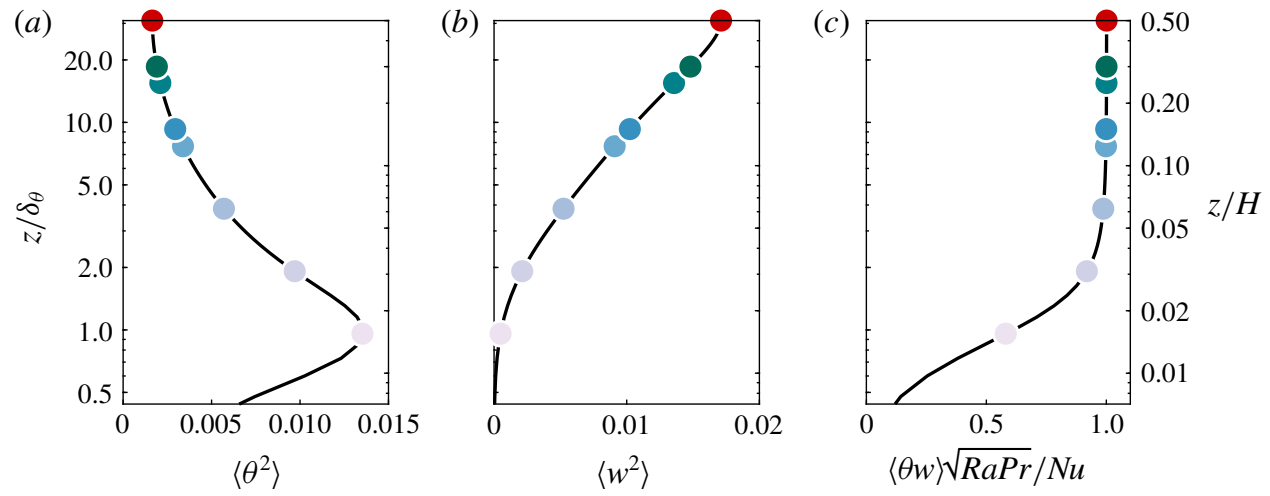

FIGURE 3. Wall-normal temperature $(a)$ and vertical-velocity $(b)$ variance profiles for $R a=$ $10^{8}$. (c) The corresponding normalized turbulent heat flux. Symbols denote the location of the spectra plotted in figure 2 with corresponding colours.

by the red lines in figure 2. Figure 2(a) shows that the temperature spectrum $k \Phi_{\theta \theta}(z / H=0.5)$ has a pronounced peak in the range $k \approx 1 \pm 0.4$ that corresponds to the superstructures (marked by a grey band as a visual aid in the figure). This peak contains approximately half of the temperature variance at $z / H=0.5$, while the remainder of the variance is spread out over a wide range of intermediate and small scales, which individually carry relatively little energy. Figure $2(b)$ reveals that the corresponding vertical-velocity spectrum $k \Phi_{w w}(z / H=0.5)$ spans approximately the same range of scales as its temperature counterpart overall. However, its shape is significantly different, as it is much more broadband and has a fairly wide peak centred around $k \approx 3.5$. It is important to note, though, that there is significant energy in the $k \Phi_{w w}$ spectrum at the scales corresponding to the thermal superstructures, which are marked by grey shading in all panels of figure 2. This implies that velocity structures of the same size as the temperature superstructures indeed exist. Yet, their contribution is overshadowed by stronger velocity fluctuations at smaller scales.

More insight into the correlation between the velocity and temperature structures is obtained by analysing the one-sided co-spectrum $\Phi_{\theta w}=\operatorname{Re}\left(\left\langle\mathcal{F}(\theta) \mathcal{F}(w)^{*}\right\rangle\right)$, where $\mathcal{F}(\cdot)$ indicates the Fourier transform in the horizontal plane and $(\cdot)^{*}$ the complex conjugate. Figure 2(c) shows that the temperature-velocity co-spectrum $k \Phi_{\theta w}$ at mid-height features a pronounced large-scale peak at $k \approx 1$. This indicates that a correlation exists between the large-scale structures in $\theta$ and $w$. Further, $k \Phi_{\theta w}(z / H=0.5)$ decreases with increasing $k$, but scales smaller than the superstructure size nevertheless contribute significantly to the turbulent heat transport. Aside from the degree of correlation between $\theta$ and $w$, their magnitudes also factor into the co-spectrum at a given scale. In order to focus on the correlation aspect only, we analyse the linear coherence spectrum

$$
\gamma_{\theta w}^{2}(k)=\frac{\left|\Phi_{\theta w}(k)\right|^{2}}{\Phi_{\theta \theta}(k) \Phi_{w w}(k)} .
$$

By definition, $0 \leqslant \gamma_{\theta w}^{2} \leqslant 1$ and the coherence may be interpreted as the square of a scale-dependent correlation coefficient. From figure $2(d)$ it is evident that $\gamma_{\theta w}^{2} \geqslant 0.8$ for almost the entire large-scale peak, with a maximum value of $\gamma_{\theta w}^{2}=0.95$ at $k=1$ 
(a)

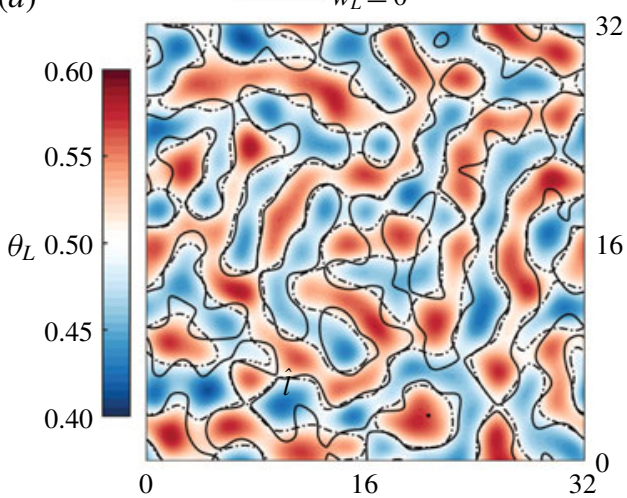

(b)

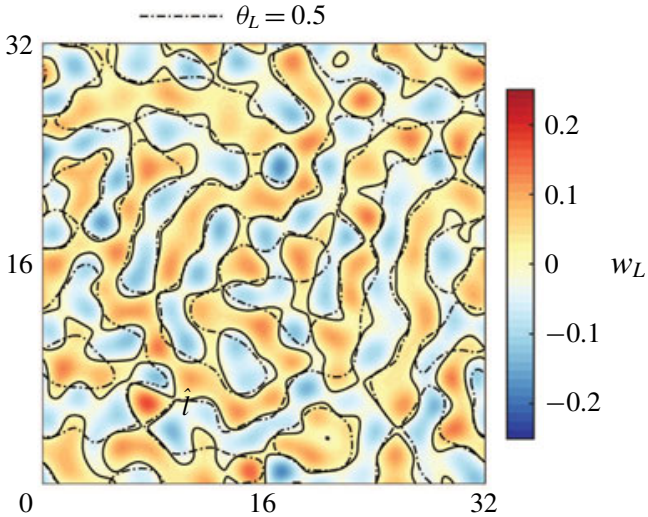

FIgURE 4. Same snapshots of temperature $(a)$ and vertical velocity $(b)$ at mid-height as presented in figure 1, but this time filtered with a spectral low-pass filter with cut-off wavenumber $k_{\text {cut }}=2.5$.

(marked by a dashed line in all panels of figure 2). The coherence quickly drops below $\gamma_{\theta w}^{2}=0.5$ for larger $k$. This explains why the overall correlation coefficient between $\theta$ and $w$, which is essentially an average over the coherence spectrum, is smaller than 0.5 as reported in Stevens et al. (2018).

In order to demonstrate also visually how well the large scales of $w$ and $\theta$ are correlated, we present the snapshots from figure 1 again in figure 4, but this time with the small-scale contributions removed. More specifically, we obtain the large-scale fields $\theta_{L}$ and $w_{L}$ using a spectral low-pass filter where the cut-off wavenumber $k_{c u t}=$ 2.5 is chosen based on the scale at which $\gamma_{\theta w}^{2}(z / H=0.5)$ drops below 0.5. Figure 4 convincingly shows that there is indeed a very good correspondence between patterns at the superstructure scale in temperature and vertical-velocity fields, not only in size but also in location.

To summarize, we have shown that patterns corresponding to the thermal superstructures also exist in the vertical velocity. For the vertical velocity, though, the contribution of the superstructures in the $k \Phi_{w w}$ spectrum is subdominant in the sense that it does not result in a spectral peak. This has previously led to the notion that the superstructures in the velocity field are smaller than in the temperature field, whereas it is really the size of the most energetic structures, as measured by the spectral peak, that is different. We will revisit the reasons for the different spectral distributions of $\theta$ and $w$ in $\S 3.2$, but we first discuss the height dependence of the trends discussed so far.

Apart from the results at mid-height, figure 2 also contains data at seven different wall-normal locations that span the full domain down to the thermal boundary layer thickness $\delta_{\theta}=1 /(2 N u)$. Remarkably, curves at all $z$-positions collapse around the peak at $k=1$ for the temperature spectra in figure $2(a)$. This suggests that there is very little evolution of the large-scale thermal structures along the vertical direction. Similarly, also the coherence between $\theta$ and $w$ (figure $2 d$ ) is almost independent of $z$ at the largest scales. In contrast, there is a pronounced increase in $k \Phi_{w w}$ around $k \approx 1$ with increasing distance away from the wall - a natural consequence of the impermeability condition at the wall. It is this increase in $k \Phi_{w w}$ that also drives a growth of the largescale peak of the co-spectrum as $z$ increases, as shown in figure $2(c)$. 
What is striking about the $k \Phi_{\theta \theta}$ spectra (figure $2 a$ ) is that at heights of the order of $\delta_{\theta}$ there exists a second strong peak in addition to the one caused by the superstructures. This small-scale peak is located at $k \approx 34$ (indicated by the dotted lines in figure 2), which corresponds to a typical small-scale structure size of approximately $11 \delta_{\theta}$. Upon comparison with figure 3(a), it becomes clear that this peak carries the energy that leads to the maximum of $\left\langle\theta^{2}\right\rangle$ at $z=\delta_{\theta}$. A similar small-scale peak is also observed for $k \Phi_{w w}$ in figure $2(b)$, even though it is located at slightly larger $k$ in this case. For $k \Phi_{w w}$, this peak broadens towards intermediate scales with increasing $z$ and the increase of $\left\langle w^{2}\right\rangle$ with increasing $z$ (see figure $3 b$ ) is mostly associated with increasing energy content at intermediate scales $k \approx 10$. It is further interesting to note that the spectral decomposition of $k \Phi_{\theta w}$ shifts from small-scale-dominated $\left(z \lesssim 3 \delta_{\theta}\right)$ over broadband $(0.1 H \lesssim z \lesssim 0.2 H)$ to a maximum at large scales for $z \gtrsim 0.2 \widetilde{H}$. At the same time, the overall heat transport $\langle\theta w\rangle$ stays approximately constant beyond $z \approx 2 \delta_{\theta}$ (see figure $3 c$ ). In connection, these observations appear consistent with the concept of merging plumes. This was advocated by, for example, Parodi et al. (2004), who found that the structure size increases going away from the wall while the flux remains constant.

\subsection{Production of temperature and vertical-velocity fluctuations}

In order to uncover the origin of the different spectral distributions of temperature and vertical velocity that became apparent in figure $2(a, b)$, we now study the variance production terms of the respective variance budgets. These production terms are (Deardorff \& Willis 1967; Kerr 2001; Togni, Cimarelli \& De Angelis 2015)

$$
S_{\theta}=-2\langle\theta w\rangle \frac{\mathrm{d} \Theta}{\mathrm{d} z}
$$

for $\left\langle\theta^{2}\right\rangle$ and

$$
S_{w}=\langle\theta w\rangle
$$

for $\left\langle w^{2}\right\rangle$. A trivial but nevertheless important implication that arises from comparing (3.3) and (3.4) is that $\langle\theta w\rangle$ generates $w$ variance directly, while temperature variance is only produced in the presence of a mean gradient $\mathrm{d} \Theta / \mathrm{d} z$. Consequently, $S_{\theta}>0$ is restricted to the thermal boundary layer $\left(z \lesssim \delta_{\theta}\right)$ since a significant mean temperature gradient exists only there. This close to the wall $\langle\theta w\rangle$ is predominantly a small-scale quantity as evidenced by $k \Phi_{\theta w}\left(z=\delta_{\theta}\right)$ in figure $2(c)$, such that $S_{\theta}$ is localized not only in space but also in scale. On the contrary, $\langle\theta w\rangle$ is almost independent of $z$ outside of the thermal boundary layer; see figure $3(c)$. Hence, also $S_{w}$ is widely distributed across the bulk of the flow. To better understand the spectral distribution of $S_{w}$, we present the data from figure $2(c)$ in cumulative form in figure 5. This figure reveals that, even at $z / H=0.5$, the large-scale peak of $\Phi_{\theta w}$ only contributes approximately $30 \%$ of the total flux $\langle\theta w\rangle$. In the central region of the flow, the bulk of the $\left\langle w^{2}\right\rangle$ production occurs at intermediate scales (say $2 \lesssim k \lesssim 10$ ). This coincides with the scales at which $k \Phi_{w w}$ peaks at these wall distances; see figure $2(b)$. This further explains why the superstructure contribution is not reflected as a spectral peak in the $k \Phi_{w w}$ spectrum.

While the analysis of the production terms provides essential insight into the reasons for the different spectral decompositions of $\left\langle\theta^{2}\right\rangle$ and $\left\langle w^{2}\right\rangle$, other aspects cannot be addressed on this basis alone. Specifically, understanding the apparently very efficient organization of small-scale temperature fluctuations into thermal superstructures 

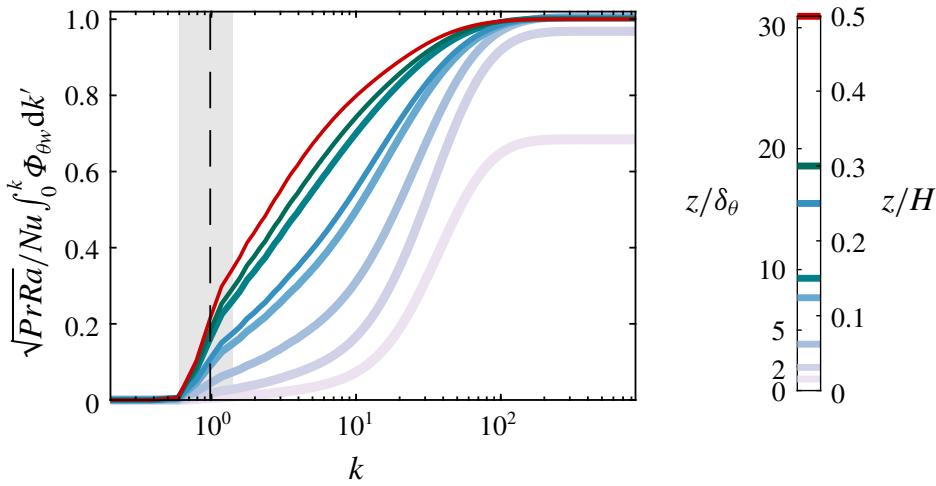

FIgURE 5. Cumulative co-spectrum $\int_{0}^{k} \Phi_{\theta w} \mathrm{~d} k^{\prime}$ for $R a=10^{8}$. The corresponding co-spectra $k \Phi_{\theta w}$ are shown in figure $2(c)$. The normalization is chosen such that the spectra add up to the relative contribution of turbulent transport to $N u$ at each wall height.

requires the analysis of inter-scale energy transfer. Such an undertaking is beyond the scope of the present work. We note, however, that an inverse (i.e. from smaller to larger scales) energy transfer is indeed observed in certain regions of the flow for both velocity and temperature when horizontally averaged budgets are considered (see e.g. Togni et al. 2015; Green et al. 2020).

\subsection{Wall-normal coherence of superstructures}

So far, we have only considered the correlation between vertical velocity and temperature at a given wall-normal location. Another important aspect is the wall-normal coherence of superstructures. There exists qualitative evidence from comparing snapshots at different heights (Stevens et al. 2018) all the way down to the skin friction field (Pandey et al. 2018) that an imprint of the large-scale structures is visible in the boundary layers. To corroborate these findings in a more systematic and quantitative manner, we again turn to the linear coherence spectrum. However, this time we do not evaluate coherence between different fields, but now we evaluate the same fields at different heights $z$ and $z_{R}$ according to

$$
\gamma_{\psi \psi}^{2}\left(z_{R} ; z, k\right)=\frac{\left|\left\langle\mathcal{F}\left(\psi\left(z_{R}\right)\right) \mathcal{F}(\psi(z))^{*}\right\rangle\right|^{2}}{\Phi_{\psi \psi}\left(z_{R}, k\right) \Phi_{\psi \psi}(z ; k)} .
$$

We fix the reference height at $z_{R}=\delta_{\theta}$. Consequently, $\gamma_{\psi \psi}^{2}\left(z_{R}=\delta_{\theta} ; z, k\right)$ is a measure of how correlated structures in field $\psi$ at scale $k$ and height $z$ are with fluctuations of the same scale at boundary layer height of the same field.

Results are presented for temperature and vertical velocity in figures $6(a)$ and $6(b)$, respectively. By definition, the result at $z=\delta_{\theta}$ is the correlation with the reference itself and therefore $\gamma_{\psi \psi}^{2}\left(z_{R}=\delta_{\theta} ; \delta_{\theta}, k\right)=1$ trivially. In the superstructure peak ( $k=1 \pm 0.4$, marked by grey shading in figure 6) $\gamma_{\psi \psi}^{2}\left(z_{R}=\delta_{\theta} ; z, k\right)$ is close to one, even at mid-height. This holds for both temperature and vertical velocity and implies a very strong degree of spatial coherence for the largest structures in both fields. Differences between $\theta$ and $w$ only occur at smaller scales. Beyond $z=2 \delta_{\theta}$ the 

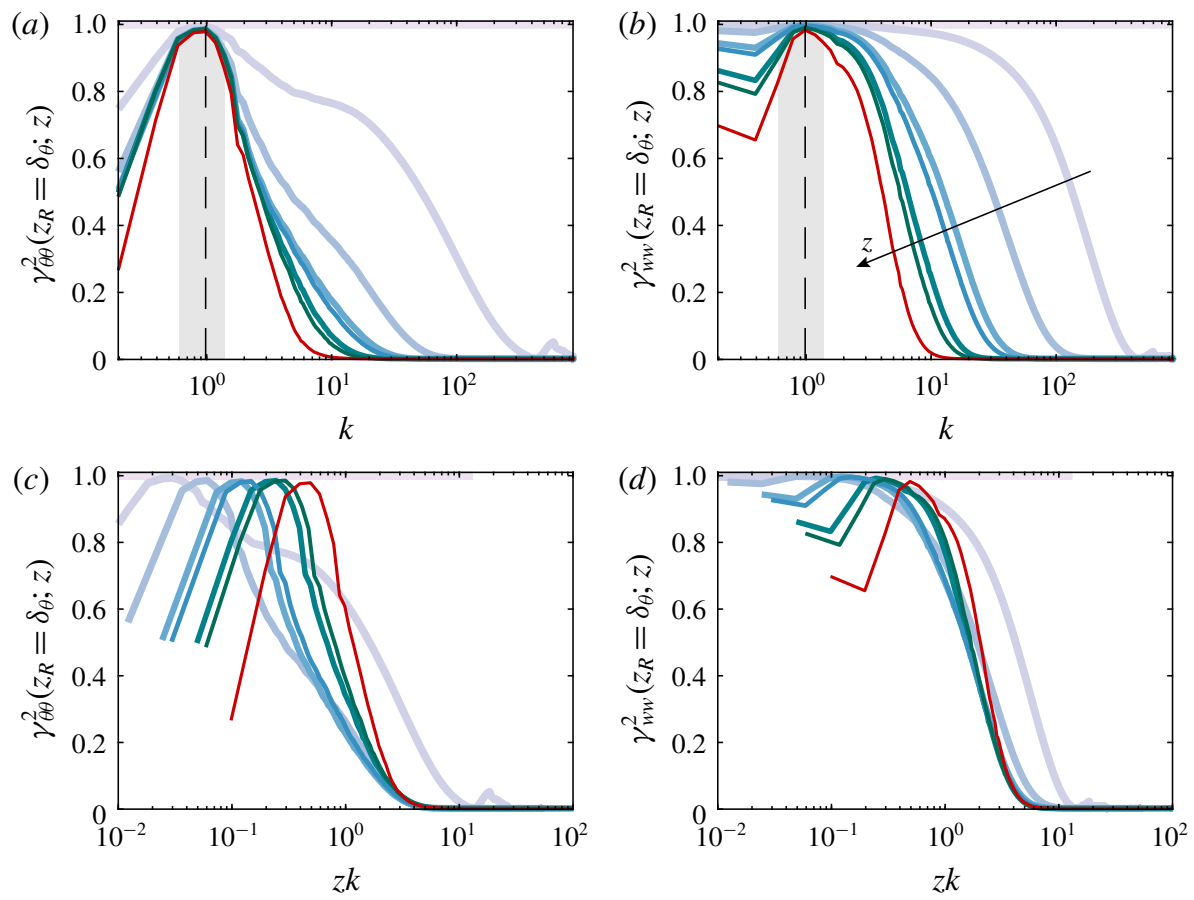

$z / H$

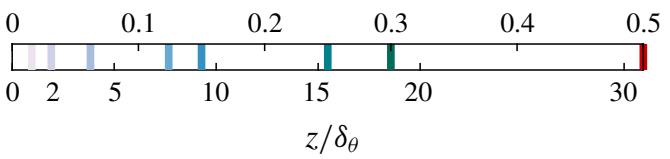

FIGURE 6. Spatial coherence spectra of temperature $(a)$ and vertical velocity $(b)$ with the reference plane at $z_{R}=\delta_{\theta}$. The data from panels $(a)$ and $(b)$ are plotted again in $(c)$ and $(d)$, respectively, as a function of $z k$ instead of $k$. All results shown are for $R a=10^{8}$.

spatial coherence of $\theta$ decreases very quickly as a function of $k$, but has a limited $z$ dependence. In contrast, curves for $\gamma_{w w}^{2}$ in figure $6(b)$ show significant variation with $z$, with the decline occurring at progressively smaller $k$ with increasing $z$. Apart from quantifying the correlation, $\gamma_{\psi \psi}^{2}\left(z_{R}=\delta_{\theta}\right)$ also provides information about the self-similarity of structures that are connected or 'attached' to the thermal boundary layer. This is of interest, since previous authors (e.g. Ahlers, Bodenschatz \& He 2014; He et al. 2014) have referred to the attached-eddy framework (Townsend 1976; Perry \& Chong 1982; Marusic \& Monty 2019), which assumes the existence of self-similar wall-attached structures, in the interpretation of their results. For the coherence spectrum, self-similarity implies that curves of $\gamma_{\psi \psi}^{2}\left(z_{R}=\delta_{\theta}\right)$ should collapse if plotted against $z k$, that is, if the scale is normalized by the distance from the wall (see Baars, Hutchins \& Marusic 2017; Krug et al. 2019). We test this for temperature and vertical velocity in figure $6(c, d)$. Clearly, self-similarity is not observed for the temperature (figure $6 c$ ). However, figure $6(d)$ shows that the data for $w$ indeed collapse to a reasonable degree for $3 \delta_{\theta} \lesssim z \lesssim 0.3 H$.

To check if self-similarity scaling in this range is a property of the velocity field in general, we additionally present results for $\gamma_{v v}^{2}\left(z_{R}=\delta_{\theta}\right)$, where $v$ is the 


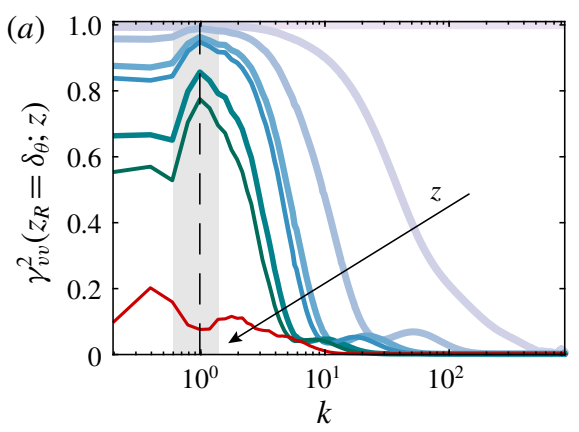

(b)

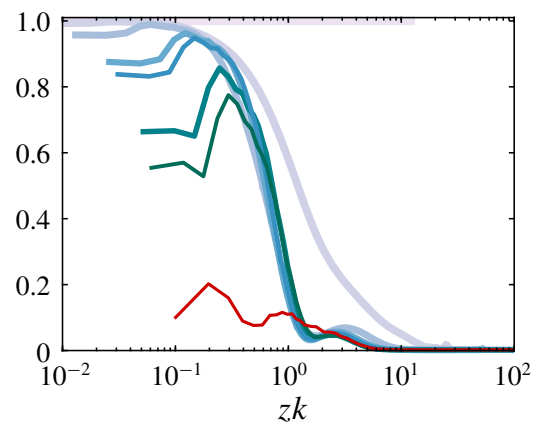

$z / H$

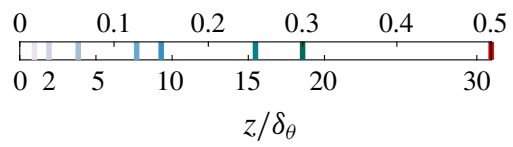

FIGURE 7. Spatial coherence spectra of horizontal velocity $v$ as a function of $(a) k$ and (b) $z k$. The reference height is $z_{R}=\delta_{\theta}$ and $R a=10^{8}$.

horizontal velocity component, in figure 7. The vertical coherence of $v$ also exhibits the same superstructure peak as observed for the other quantities, which can be seen in figure 7(a). Only its magnitude decreases with increasing $z$ and is close to zero at mid-height. This is consistent with the roll structures not having a horizontal component at $z / H \approx 0.5$ and also the spectral energy $\Phi_{v v}(k=1 \pm 0.4)$ (not shown) is minimal there. As figure $7(b)$ shows, $\gamma_{v v}^{2}\left(z_{R}=\delta_{\theta}\right)$ displays the same collapse when plotted versus $z k$ and in the same range of $z$ as previously observed for $w$. This means that for the velocity fields in a significant part of the domain (at least $3 \delta_{\theta} \lesssim z \lesssim 0.3 H$ ) structures attached to the boundary layer display self-similar behaviour. The same trends are observed at different $R a$ but are not shown here for brevity.

\subsection{Rayleigh-number trends}

As a final point, we study the $R a$ dependence of the properties discussed in $\S 3.1$. To this end, we present results for $\gamma_{\theta w}^{2}$ evaluated at mid-height for $10^{5} \leqslant R a \leqslant 10^{9}$ in figure $8(a)$. The magnitude and the shape of the large-scale peak are nearly independent of $R a$. However, the peak location shifts towards smaller $k$ with increasing $R a$. The corresponding increase in the large-scale structure is quantified in figure $8(b)$, where the triangles indicate the structure size $(\hat{l}=2 \pi / \hat{k})$ corresponding to the peak in the coherence. Here, the peak location $\hat{k}$ is obtained from fitting a parabola to three points centred around the peak of $\gamma_{\theta w}^{2}$ and the results are also listed in table 1 . Evidently, $\hat{l}$ is significantly larger than the wavelength of the structure at the onset of convection, which is $\approx 2$ (e.g. Drazin \& Reid 2004). Additionally, length scales corresponding to the spectral peak in $\Phi_{\theta \theta}$ (circles), $\Phi_{w w}$ (filled squares) and $k \Phi_{w w}$ (open squares) are included in figure $8(b)$ and the corresponding spectra are shown in figure $8(c, d)$. The spectral peak from the temperature spectrum corresponds to slightly larger length scales compared to the results based on $\gamma_{\theta w}^{2}$, but the differences are quite small. Owing to its broadband nature, the spectral peaks for the vertical velocity are found at different locations in the regular and premultiplied spectra. The 

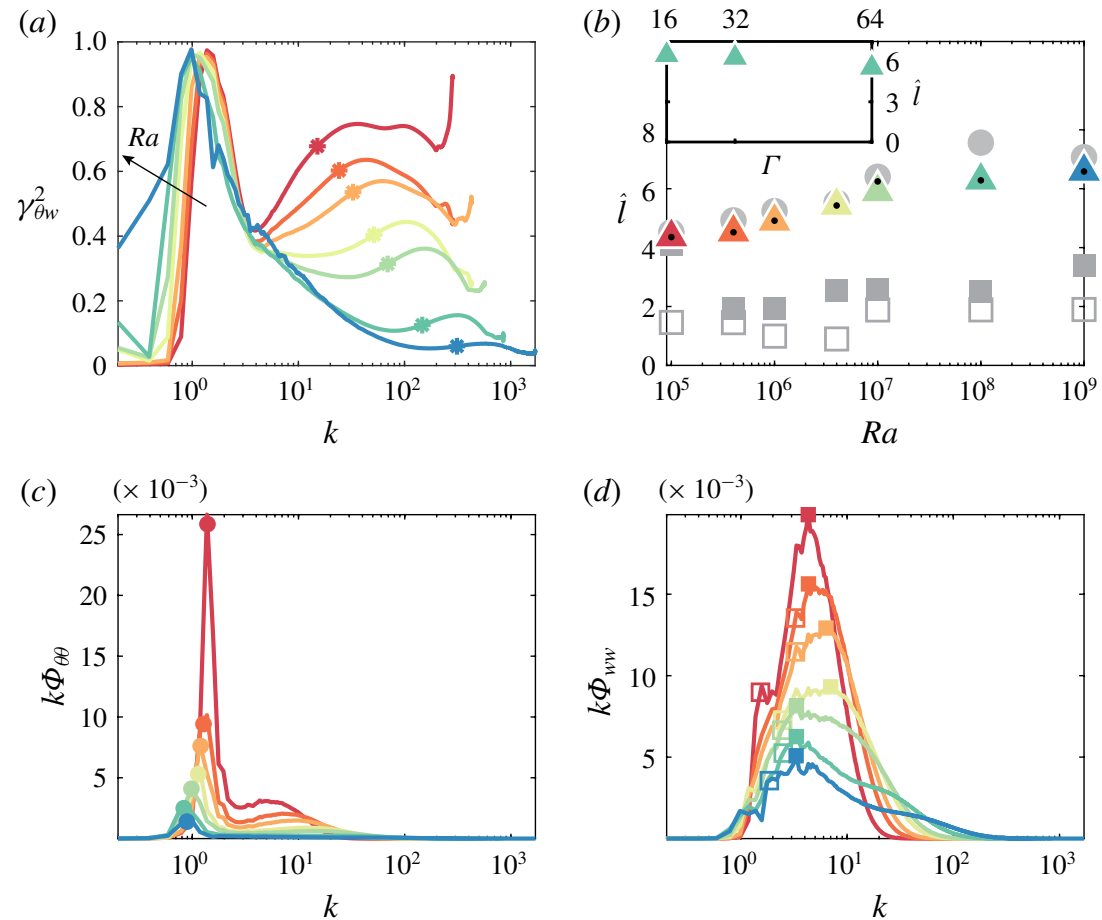

Figure 8. (a) Coherence spectrum at mid-height for $10^{5} \leqslant R a \leqslant 10^{9}$; see panel $(b)$ for the colour code. Stars indicate the wavenumber corresponding to $10 \eta$ at the respective $R a$. (b) Wavelength $\hat{l}$ of the spectral peaks of $\gamma_{\theta w}^{2}$ (triangles), $\Phi_{\theta \theta}$ (circles), $\Phi_{w w}$ (filled squares), $k \Phi_{w w}$ (open squares) and $k \Phi_{\theta w}$ (black dots). The corresponding spectra $k \Phi_{\theta \theta}$ and $k \Phi_{w w}$ are shown in panels $(c)$ and $(d)$ with symbols marking the peak locations as described for panel $(b)$. The inset in panel $(b)$ additionally shows the aspect-ratio dependence of $\hat{l}$ based on $\gamma_{\theta w}^{2}$ at $R a=10^{8}$; see the Appendix for details.

maximum of $\Phi_{w w}$ only agrees with the results based on coherence and temperature at $R a=10^{5}$. For higher $R a$ fluctuations at intermediate length scales dominate the velocity spectrum. Therefore, the use of the velocity spectra leads to significantly lower estimates for $\hat{l}$ than the temperature spectra at higher $R a$, as mentioned in $\S 3.1$.

The dependence of the superstructure size on the aspect ratio $\Gamma$ of the periodic domain was already discussed in Stevens et al. (2018). From their results, it appears that the superstructure size based on the peak in $\Phi_{\theta \theta}$ increases monotonically (albeit slowly for $\Gamma>16$ ) with increasing $\Gamma$. The inset of figure $8(b)$ shows that $\hat{l}$ based on $\gamma_{\theta w}^{2}$ decreases slightly if $\Gamma$ is increased from 16 to 64 . This difference is not rooted in the fact that a different metric is employed here, but is caused by an error in the computation of the spectra presented in Stevens et al. (2018). We plot the recomputed spectra in the Appendix and these show that the temperature spectral peak indeed exhibits the same trend as the one observed for $\gamma_{\theta w}^{2}$.

The concept of a convection roll, i.e. a thermally driven velocity structure, suggests a definition of the superstructure size in $\mathrm{RBC}$ as the scale where the correlation between temperature and velocity fields is maximum. We therefore argue that 
conceptually the most straightforward way to define the superstructure size is via the coherence spectrum. It should be noted that the coherence peak is not necessarily coincident with the peak of the co-spectrum due to the different distributions of $\Phi_{\theta \theta}$ and $\Phi_{w w}$. In practice, however, the peaks of $\gamma_{\theta w}^{2}$ and $k \Phi_{\theta w}$ coincide within measurement accuracy for the cases presented here (see figure $8 b$ ). This seems to be a consequence of the sharp drop-off of $\gamma_{\theta w}^{2}$ and $\Phi_{\theta \theta}$ with increasing $k$ which outweighs the increase in $\Phi_{w w}$. The situation may change, however, e.g. for different $\operatorname{Pr}$ numbers. Some caution is therefore advised in this matter. For a case in point, we note that the small-scale peak in $k \Phi_{\theta w}$ at $z=\delta_{\theta}$ (figure $2 c$ ) is without counterpart in $\gamma_{\theta w}^{2}$ (figure $2 c$ ). This indicates that the peak in turbulent transport is predominantly driven by magnitude, not coherence. The peaks of the $\Phi_{w w}$ and the $k \Phi_{w w}$ spectra may be misleading as indicators for superstructure size since the velocity spectra are dominated by motions at intermediate length scales.

As an aside, we discuss the increase of $\gamma_{\theta w}^{2}$ that is seen to occur at high $k$ in figure $8(a)$. This increase at small scales occurs for lower values of $k$ and is stronger for lower $R a$. A comparison with figure $8(c, d)$ reveals that there is only minimal energy at these small scales. These observations are consistent with the notion that the higher values of $\gamma_{\theta w}^{2}$ mark the transition to a viscous-dominated regime. In the viscous regime, the correlation between $\theta$ and $w$ is high because the balance is predominantly between buoyancy and viscous forces. This is similar to the situation at the onset of convection, where the correlation between velocity and temperature fluctuations is very high (Bodenschatz, Pesch \& Ahlers 2000). To lend support to this understanding, we added the length scale $10 \eta$ as a reference scale for the viscous regime in figure 8. Here, $\eta=\left(v^{3} /\langle\varepsilon\rangle_{V}\right)^{1 / 4}$ is the Kolmogorov length scale and $\langle\varepsilon\rangle_{V}$ is the volume-averaged dissipation rate obtained from the identity $\langle\varepsilon\rangle_{V}=(N u-1) / \sqrt{\operatorname{RaPr}}$. It is seen in figure $8(a)$ that the scale at which the high-wavenumber increase of $\gamma_{\theta w}^{2}$ occurs roughly coincides with $10 \eta$ for $R a>10^{6}$, just as expected from the above. The agreement is less good for the (marginally turbulent) cases at even lower $R a$, where the increase in $\gamma_{\theta w}^{2}$ starts at scales significantly larger than $10 \eta$.

The $R a$ dependence of the near-wall characteristics of the temperature field are displayed in figure 9 in which $k \Phi_{\theta \theta}$ is plotted at $z=\delta_{\theta}$ for each $R a$. This figure shows that the small-scale peak contributes an increasingly larger part of the total energy with increasing $R a$. At the same time, the scale separation between the small scales and the large-scale superstructures increases with increasing thermal driving. We define the length scale of the small-scale structures as $\hat{l}_{\delta}=2 \pi / \hat{k}_{\delta}$, where $\hat{k}_{\delta}$ is the location of the high- $k$ peak. Figure $9(b)$ shows that $\hat{l}_{\delta}$ is approximately constant for $10^{5} \leqslant R a \leqslant 10^{9}$, when normalized with the boundary layer thickness $\delta_{\theta}$. The magnitude of the ratio $\hat{l}_{\delta} / \delta_{\theta}$ differs slightly depending on the quantity considered. The most energetic small-scale structures for the temperature are approximately $11.5 \delta_{\theta}$, for $w$ it is approximately $8.5 \delta_{\theta}$, and $k \Phi_{\theta w}$ peaks at approximately $10 \delta_{\theta}$.

\section{Conclusion}

Contrary to what prior analysis (Pandey et al. 2018; Stevens et al. 2018) appeared to suggest, we found that superstructures of approximately the same size exist in the temperature and vertical-velocity fields in large-aspect-ratio Rayleigh-Bénard flow. These result in a very significant large-scale peak in the linear coherence spectrum of $\theta$ and $w$ that signifies almost perfect correlation at the large length scales. Unlike the case for $\theta$, we find that the superstructures in $w$ do not correspond to a spectral peak 

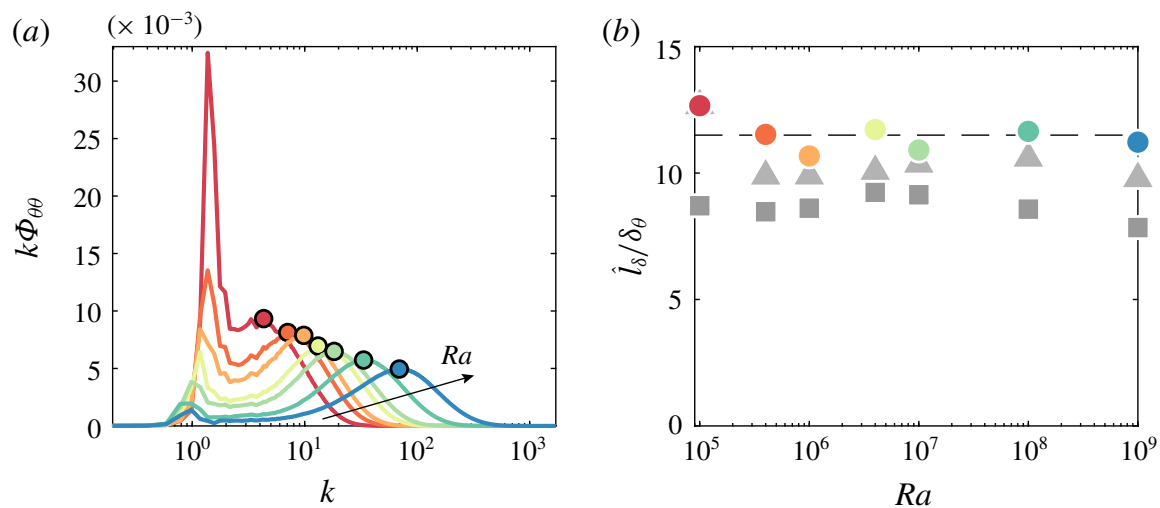

Figure 9. (a) Premultiplied temperature power spectra $k \Phi_{\theta \theta}$ at $z=\delta_{\theta}$ for $10^{5} \leqslant R a \leqslant 10^{9}$. Symbols mark the location of the small-scale peak determined as the maximum of $k \Phi_{\theta \theta}(k)$ for $k>2$. Note that, at the lower $R a$, this peak does not correspond to a global maximum of $k \Phi_{\theta \theta}$. (b) Length scale $\hat{l}_{\delta}$ associated with the small-scale peak of $k \Phi_{\theta \theta}$ (circles), $k \Phi_{w w}$ (squares) and $k \Phi_{\theta w}$ (triangles) normalized with the thermal boundary layer thickness $\delta_{\theta}$. The dashed line is at $\mathbf{1 1 . 5}$ for reference.

in the power spectrum of $w$. This difference has previously led to the above-mentioned confusion regarding potentially different sizes of the largest structures in $\theta$ and $w$. The fact that the most energetic motions, as measured by the peak in the spectra, occur at intermediate scales for $w$, but at the superstructure scale for $\theta$, can be explained by differences in the production terms of the respective variance budgets. In particular, temperature production is confined to the boundary layer and small scales, while buoyancy forcing acts at intermediate scales and throughout the entire bulk of the flow. Furthermore, we find that the superstructure scale increases with $R a$ for $10^{5} \leqslant R a \leqslant 10^{9}$, i.e. the full range investigated here, when the structure size is based on the coherence spectrum as suggested. It should be noted that integral length scales of temperature and turbulent kinetic energy as used in Stevens et al. (2018) do not accurately capture this growth, which shows the importance of selecting the appropriate metric to quantify superstructures in RBC.

In agreement with previous observations of superstructure footprints in the boundary layer region, we find an almost perfect spatial correlation of the superstructure scales from the boundary layer height $\delta_{\theta}$ up to mid-height, for both $\theta$ and $w$. Also, the temperature spectra, as well as $\gamma_{\theta w}^{2}$, are seen to collapse at different heights. Hence, there is no noticeable dependence of the superstructure scale on $z$, effectively ruling out a significant growth of the thermal structures due to horizontal transport while they are travelling upward as was suggested by Pandey et al. (2018). The decrease of spatial correlation (quantified by the linear coherence spectrum) at intermediate scales when increasing the distance to the reference height $\delta_{\theta}$ is observed to follow a self-similar trend for $w$ and the horizontal component $v$, but not for $\theta$. The reason for this difference remains unclear but warrants further investigation.

Moreover, we find that the energy distribution of the temperature field is bimodal. Besides the $z$-independent large-scale contribution of the superstructures, premultiplied spectra reveal the existence of a pronounced small-scale peak at boundary layer height. The two peaks are separated by a spectral gap that increases with $R a$, which is also 
visible in the co-spectra of $\theta$ and $w$. However, $k \Phi_{w w}$ displays a small-scale peak only near the wall and is broadband otherwise. For the temperature fluctuations, the small-scale peak carries the energy that leads to the maximum of $\left\langle\theta^{2}\right\rangle$ at $z=\delta_{\theta}$ (see e.g. Wang, He \& Tong (2016)). The length scale of the associated structures is approximately $l_{\delta} \approx 10 \delta_{\theta}$. It is interesting to note that the situation described here bears a close resemblance to findings in turbulent boundary layers. There, an 'inner peak' is observed that is fixed at 15 viscous units $\left(l_{\text {visc }}\right)$ away from the wall and with typical streamwise length scales of approximately $1000 l_{\text {visc }}$ (Hutchins \& Marusic 2007b). The scale separation between the 'inner peak' and the large-scale structures is, however, significantly stronger in RBC. This appears to suggest distinctly different processes, as was already pointed out in Pandey et al. (2018) in a different context, and raises questions about their interaction. A better understanding of these aspects will be very insightful for modelling approaches.

\section{Acknowledgements}

The authors acknowledge stimulating discussions with W. Baars and thank A. Blass for help with the data. This work is supported by the Twente Max-Planck Center, the German Science Foundation (DFG) via program SSP 1881, and the ERC (European Research Council) Starting Grant no. 804283 UltimateRB. The authors gratefully acknowledge the Gauss Centre for Supercomputing e.V. (www.gauss-centre.eu) for funding this project by providing computing time on the GCS Supercomputer SuperMUC-NG at Leibniz Supercomputing Centre (www.lrz.de). Part of the work was carried out on the national e-infrastructure of SURFsara, a subsidiary of SURF cooperation, the collaborative ICT organization for Dutch education and research.

\section{Declaration of interests}

The authors report no conflict of interest.

\section{Appendix. Aspect-ratio dependence of the superstructure size}

The aspect-ratio dependence of the superstructures has been studied in Stevens et al. (2018) before. However, there was a bug in the radial averaging of the spectra, which led to a faulty spectral distribution, especially at the largest scales. In figure 10, we present results at $R a=10^{8}$ for aspect ratios varying between 3 and 64 . Up to $\Gamma=8$ there is no clear peak, as the highest values are attained for the smallest $k$ for all quantities displayed. Distinct large-scale peaks emerge for $\Gamma \geqslant 16$ for $k \Phi_{\theta \theta}$ (figure 10a), $k \Phi_{\theta w}$ (figure 10c) and $\gamma_{\theta w}^{2}$ (figure 10d). The locations of these peaks (again obtained by fitting a parabola over three points) are shown in figure 10. Results based on $k \Phi_{\theta \theta}$ and $\gamma_{\theta w}^{2}$ decrease monotonically with increasing $\Gamma$, while $\hat{l}$ based on the co-spectrum increases between $\Gamma=16$ and $\Gamma=32$. The peaks in $k \Phi_{w w}$, and remarkably these spectra in general, exhibit only a minor sensitivity to $\Gamma$. The peaks of the velocity spectra remain, however, at intermediate scales significantly smaller than the superstructure size.

The conclusion of Stevens et al. (2018) that very large domains are needed to fully converge the superstructure size remains valid, even though, at least for the values checked here, the trend is opposite (decreasing size) to what was previously believed. The scale $\hat{l}$ still varies by approximately $10 \%$ between the cases with $\Gamma=32$ and $\Gamma=64$. The more basic requirement is, however, that $\Gamma \gtrsim 16$ because only then is the large-scale peak actually resolved. 

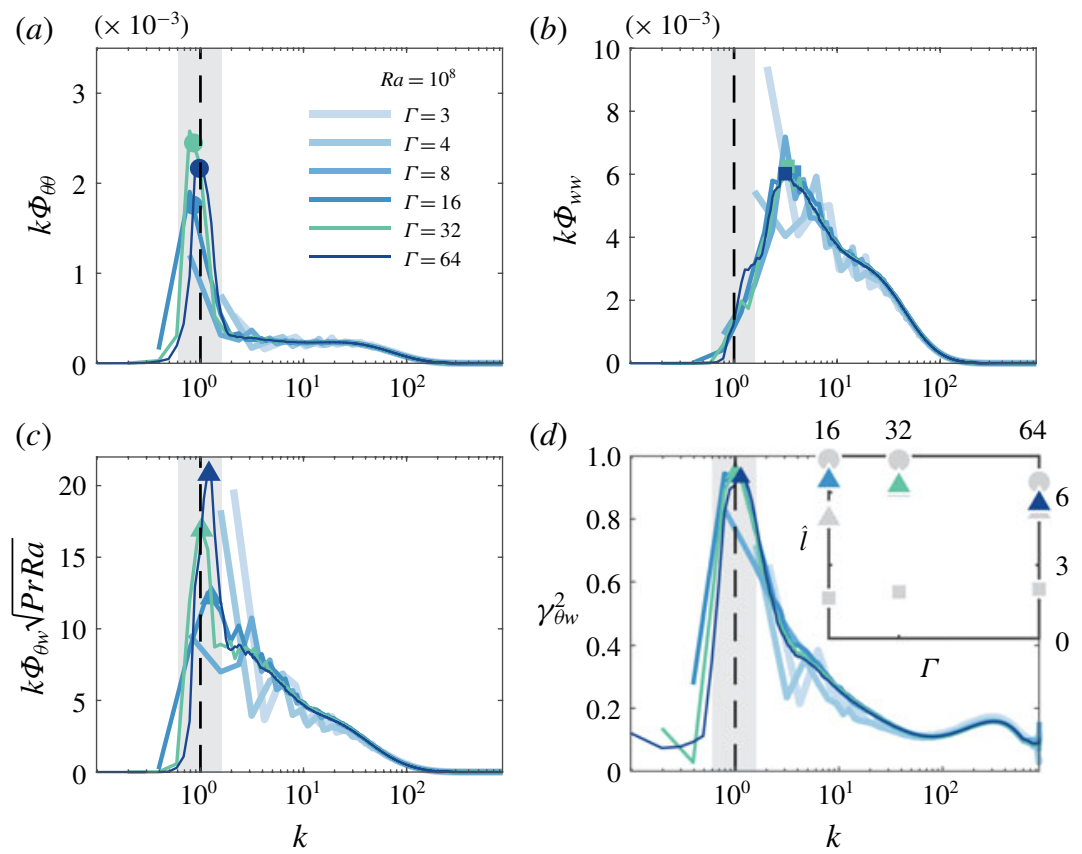

FIgURE 10. Premultiplied temperature $(a)$ and vertical-velocity $(b)$ power spectra. The premultiplied co-spectrum $k \Phi_{\theta w}(c)$ is normalized such that it integrates to the turbulent heat flux. $(d)$ Linear coherence spectrum $\gamma_{\theta w}^{2}$. Symbols indicate the peak values for the three largest $\Gamma$. The inset shows $\hat{l}$ based on coherence (coloured triangles) and co-spectra (grey triangles), as well as on temperature (circles) and velocity (squares) power spectra. The dashed vertical line indicates $k=1$ and the grey shaded area marks the approximate range of superstructure scales $k=1 \pm 0.4$ (same as in figure 2). All results presented here are computed for $R a=10^{8}$ at mid-height; the legend in $(a)$ applies to all panels.

\section{REFERENCES}

Ahlers, G., Bodenschatz, E. \& He, X. 2014 Logarithmic temperature profiles of turbulent Rayleigh-Bénard convection in the classical and ultimate state for a Prandtl number of 0.8 . J. Fluid Mech. 758, 436-467.

Ahlers, G., Grossmann, S. \& Lohse, D. 2009 Heat transfer and large scale dynamics in turbulent Rayleigh-Bénard convection. Rev. Mod. Phys. 81 (2), 503-537.

BAARs, W. J., Hutchins, N. \& MARUSic, I. 2017 Self-similarity of wall-attached turbulence in boundary layers. J. Fluid Mech. 823, R2.

BAilon-Cuba, J., EMran, M. S. \& SChUMACHER, J. 2010 Aspect ratio dependence of heat transfer and large-scale flow in turbulent convection. J. Fluid Mech. 655, 152-173.

Bodenschatz, E., Pesch, W. \& Ahlers, G. 2000 Recent developments in Rayleigh-Bénard convection. Annu. Rev. Fluid Mech. 32 (1), 709-778.

Chillà, F. \& SChumacher, J. 2012 New perspectives in turbulent Rayleigh-Bénard convection. Eur. Phys. J. E 35 (7), 58.

Cierpka, C., Kästner, C., Resagk, C. \& Schumacher, J. 2019 On the challenges for reliable measurements of convection in large aspect ratio Rayleigh-Bénard cells in air and sulfurhexafluoride. Exp. Therm. Fluid Sci. 109, 109841. 
Deardorff, J. W. \& Willis, G. E. 1967 Investigation of turbulent thermal convection between horizontal plates. J. Fluid Mech. 28 (4), 675-704.

Drazin, P. G. \& REID, W. H. 2004 Hydrodynamic Stability. Cambridge University Press.

Du Puits, R., Resagk, C. \& Thess, A. 2013 Thermal boundary layers in turbulent Rayleigh-Bénard convection at aspect ratios between 1 and 9. New J. Phys. 15 (1), 013040.

EmRan, M. S. \& Schumacher, J. 2015 Large-scale mean patterns in turbulent convection. J. Fluid Mech. 776, 96-108.

FitzJarRald, D. E. 1976 An experimental study of turbulent convection in air. J. Fluid Mech. 73 (4), 693-719.

Green, G., Vlaykov, D. G., Mellado, J. P. \& Wilczek, M. 2020 Resolved energy budget of superstructures in Rayleigh-Bénard convection. J. Fluid Mech. (in press) doi:10.1017/jfm.2019.1008.

Hartlep, T., Tilgner, A. \& Busse, F. H. 2003 Large scale structures in Rayleigh-Bénard convection at high Rayleigh numbers. Phys. Rev. Lett. 91 (6), 064501.

Hartlep, T., Tilgner, A. \& Busse, F. H. 2005 Transition to turbulent convection in a fluid layer heated from below at moderate aspect ratio. J. Fluid Mech. 544, 309-322.

He, X., van Gils, D. P. M., Bodenschatz, E. \& Ahlers, G. 2014 Logarithmic spatial variations and universal $f^{-1}$ power spectra of temperature fluctuations in turbulent Rayleigh-Bénard convection. Phys. Rev. Lett. 112 (17), 174501.

HogG, J. \& Ahlers, G. 2013 Reynolds-number measurements for low-Prandtl-number turbulent convection of large-aspect-ratio samples. J. Fluid Mech. 725, 664-680.

Huisman, S. G., Van Der Veen, R. C., Sun, C. \& Lohse, D. 2014 Multiple states in highly turbulent Taylor-Couette flow. Nat. Commun. 5, 3820.

Hutchins, N. \& MARUSIC, I. $2007 a$ Evidence of very long meandering features in the logarithmic region of turbulent boundary layers. J. Fluid Mech. 579, 1-28.

Hutchins, N. \& MARUSic, I. $2007 b$ Large-scale influences in near-wall turbulence. Phil. Trans. R. Soc. Lond. A 365 (1852), 647-664.

Kerr, R. M. 2001 Energy budget in Rayleigh-Bénard convection. Phys. Rev. Lett. 87 (24), 244502.

Krug, D., BAars, W. J., Hutchins, N. \& Marusic, I. 2019 Vertical coherence of turbulence in the atmospheric surface layer: connecting the hypotheses of Townsend and Davenport. Boundary-Layer Meteorol. 172 (2), 199-214.

Lee, M. \& Moser, R. D. 2018 Extreme-scale motions in turbulent plane Couette flows. J. Fluid Mech. 842, 128-145.

Lohse, D. \& XIA, K.-Q. 2010 Small-scale properties of turbulent Rayleigh-Bénard convection. Annu. Rev. Fluid Mech. 42, 335-364.

Marusic, I. \& Monty, J. P. 2019 Attached eddy model of wall turbulence. Annu. Rev. Fluid Mech. 51, 49-74.

Morris, S. W., Bodenschatz, E., Cannell, D. S. \& Ahlers, G. 1993 Spiral defect chaos in large aspect ratio Rayleigh-Bénard convection. Phys. Rev. Lett. 71 (13), 2026.

Pandey, A., Scheel, J. D. \& SChumacher, J. 2018 Turbulent superstructures in Rayleigh-Bénard convection. Nat. Commun. 9 (1), 2118.

Parodi, A., von Hardenberg, J., Passoni, G., Provenzale, A. \& Spiegel, E. A. 2004 Clustering of plumes in turbulent convection. Phys. Rev. Lett. 92 (19), 194503.

Perry, A. E. \& ChOng, M. S. 1982 On the mechanism of wall turbulence. J. Fluid Mech. 119, 173-217.

Van Der Poel, E. P., Ostilla-Mónico, R., Donners, J. \& Verzicco, R. 2015 A pencil distributed finite difference code for strongly turbulent wall-bounded flows. Comput. Fluids 116, 10-16.

SAKievich, P. J., Peet, Y. T. \& Adrian, R. J. 2016 Large-scale thermal motions of turbulent Rayleigh-Bénard convection in a wide aspect-ratio cylindrical domain. Intl J. Heat Mass Transfer 61, 183-196.

Shishinana, O., Stevens, R. J. A. M., Grossmann, S. \& Lohse, D. 2010 Boundary layer structure in turbulent thermal convection and its consequences for the required numerical resolution. New J. Phys. 12, 075022. 
Shishinina, O. \& WAgneR, C. 2005 A fourth order accurate finite volume scheme for numerical simulations of turbulent Rayleigh-Bénard convection in cylindrical containers. C. R. Méc 333, $17-28$.

Shishina, O. \& WAgner, C. 2006 Analysis of thermal dissipation rates in turbulent RayleighBénard convection. J. Fluid Mech. 546, 51-60.

Shishinina, O. \& WAgner, C. 2007 Local heat fluxes in turbulent Rayleigh-Bénard convection. Phys. Fluids 19, 085107.

Stevens, R. J. A. M., Blass, A., Zhu, X., Verzicco, R. \& Lohse, D. 2018 Turbulent thermal superstructures in Rayleigh-Bénard convection. Phys. Rev. Fluids 3 (4), 041501.

Stevens, R. J. A. M., Lohse, D. \& Verzicco, R. 2011 Prandtl and Rayleigh number dependence of heat transport in high Rayleigh number thermal convection. J. Fluid Mech. 688, 31-43.

Stevens, R. J. A. M., Verzicco, R. \& Lohse, D. 2010 Radial boundary layer structure and Nusselt number in Rayleigh-Bénard convection. J. Fluid Mech. 643, 495-507.

Sun, C., Ren, L.-Y., Song, H. \& XIA, K.-Q. $2005 a$ Heat transport by turbulent Rayleigh-Bénard convection in $1 \mathrm{~m}$ diameter cylindrical cells of widely varying aspect ratio. J. Fluid Mech. 542, 165-174.

Sun, C., Ren, L.-Y., Song, H. \& XiA, K.-Q. $2005 b$ Heat transport by turbulent Rayleigh-Bénard convection in $1 \mathrm{~m}$ diameter cylindrical cells of widely varying aspect ratio. J. Fluid Mech. 542, 165-174.

Togni, R., Cimarelli, A. \& De Angelis, E. 2015 Physical and scale-by-scale analysis of RayleighBénard convection. J. Fluid Mech. 782, 380-404.

Townsend, A. A. 1976 The Structure of Turbulent Shear Flow. Cambridge University Press.

Verma, M. K. 2018 Physics of Buoyant Flows: From Instabilities to Turbulence. World Scientific.

VERZICCO, R. \& ORLANDI, P. 1996 A finite-difference scheme for three-dimensional incompressible flow in cylindrical coordinates. J. Comput. Phys. 123, 402-413.

Von Hardenberg, J., Parodi, A., Passoni, G., Provenzale, A. \& Spiegel, E. A. 2008 Large-scale patterns in Rayleigh-Bénard convection. Phys. Lett. A 372 (13), 2223-2229.

WANG, Y., He, X. \& Tong, P. 2016 Boundary layer fluctuations and their effects on mean and variance temperature profiles in turbulent Rayleigh-Bénard convection. Phys. Rev. Fluids 1 (8), 082301.

Young, G. S., Kristovich, D. A. R., HJelmfelt, M. R. \& Foster, R. C. 2002 Rolls, streets, waves, and more: a review of quasi-two-dimensional structures in the atmospheric boundary layer. Bull. Am. Meteorol. Soc. 83 (7), 997-1002.

Zhou, Q., LiU, B.-F., LI, C.-M. \& ZHONG, B.-C. 2012 Aspect ratio dependence of heat transport by turbulent Rayleigh-Bénard convection in rectangular cells. J. Fluid Mech. 710, 260-276.

Zhu, X., Phillips, E., ArZa, V. S., Donners, J., Ruetsch, G., Romero, J., Ostilla-Mónico, R., YANG, Y., LOHSE, D., VerZICCO, R. et al. 2018 AFiD-GPU: a versatile Navier-Stokes solver for wall-bounded turbulent flows on GPU clusters. Comput. Phys. Commun. 229, $199-210$. 